\title{
Consultorio Farmacéutico: Un cambio de paradigma a nivel asistencial en el que se brinda atención farmacéutica directa a los usuarios de medicamentos.
}

\section{Pharmaceutical Office: A change of paradigna at the healthcare level in which it provides direct pharmaceutical care to drug users}

Pág. 10-15

Dr. Luis Carlos Monge Bogantes, MSc'.

Farmacéutico - Docente.

Recibida: 23/09/19

1Máster en Atención Farmacéutica Integral de la Universitat de Barcelona, España.

Aprobada:26/09/19

Doctor en Farmacia de la Universidad de Costa Rica,San José, Costa Rica.

Docente en la Universidad de Ciencias Médicas (UCIMED), San José, Costa Rica.

\section{RESUMEN:}

"La farmacia en los últimos años ha tenido que reinventarse no una sino en múltiples ocasiones a lo largo de los últimos siete décadas aproximadamente en las que desde la conceptualización de la farmacia clínica y el desarrollo de la farmacia hospitalaria (toxicología, información de medicamentos, farmacocinética, radiofarmacia, farmacia oncológica, preparaciones magistrales, entre otras actividades de importancia clínica) hemos llegado en los últimos veinte años al desarrollo de la nueva conceptualización de la atención farmacéutica no solo en el ámbito clínico-hospitalario sino también en el cuidado directo de los usuarios de los medicamentos a nivel asistencial privado, es por eso de gran importancia hoy en día el desarrollo de la consulta farmacéutica privada en beneficio de la calidad de vida de la población y el garantizar la seguridad en el uso racional y responsable de los medicamentos.

\section{Palabras Clave: Consulta Farmacéutica,} Atención Farmacéutica, Farmacéutico Asistencial, Seguridad del Paciente, Uso Racional de Medicamentos.

\section{ABSTRACT}

"The pharmacy in recent years has had to reinvent itself not once but on multiple occasions over the last seven decades approximately in which from the conceptualization of the clinical pharmacy and the development of the hospital pharmacy (toxicology, medication information, pharmacokinetics, radiopharmacy, oncological pharmacy, master preparations, among other activities of clinical importance) we have arrived in the last twenty years to the development of the new conceptualization of pharmaceutical care not only in the clinical-hospital field but also in the direct care of Users of medicines at the private healthcare level, it is therefore of great importance today the development of the private pharmaceutical consultation for the benefit of the population's quality of life and the safety protocol in the rational and responsible use of medicines.

Keywords: Pharmaceutical Consultation, Pharmaceutical Care, Assistance Pharmacist, Patient Safety, Rational Use of Medicines

Con el desarrollo en los Estados Unidos del concepto de Farmacia Clínica desde la década del cincuenta, en el siglo pasado; el rol del farmacéutico asistencial ha ido variando y evolucionando en muchas y distintas formas; aún que ya desde los años cuarentas en la Universidad de Washington 
en Seattle se podían ver cambios en el rumbo que hoy en día tiene la profesión farmacéutica.

"Los farmacéuticos son profesionales sanitarios específicamente capacitados e instruidos que disponen de la autorización correspondiente, para gestionar la dispensación de medicamentos a los usuarios y realicen las tareas adecuadas para garantizar la seguridad y el uso eficaz de los medicamentos". (Informe técnico: 25 de septiembre día mundia del farmacéutico., 2012)

Pasando de la formulación y la dispensación a la farmacia clínica en el año 1961 con la publicación de Francke (Francke, 1970). Como farmacia clínica se conoce al área en la que la profesión se ocupa directamente del cuidado del paciente haciendo particular énfasis en la terapia con medicamentos, donde se busca desarrollar una actividad orientada hacia el paciente.

Este desarrollo de la farmacia clínica en los ámbitos hospitalarios ha logrado desarrollar muchos servicios que hoy en día son claves como son el sistema de distribución en dosis unitarias, los centros de información de medicamentos, las unidades de farmacias satélites en los centros hospitalarios, el servicio de mezclas endovenosas, el perfil farmacoterapéutico de los pacientes, programas de alimentación parenteral, radiofarmacia, se han generado estudios sobre los errores de medicación y la seguridad del paciente, monitorización de los efectos adversos a medicamentos y hasta la localización del farmacéutico en el entorno del paciente; entre muchos otros servicios que se brindan en centros hospitalarios.

Se puede decir que la farmacia clínica se encarga de toda aquella parte de la asistencia sanitaria que promueve el uso seguro, efectivo y económico de los medicamentos por los individuos y por la sociedad (Giráldez, 1985).

Una de las funciones de los regentes farmacéuticos desde la "oficina de farmacia" o lo que conocemos en nuestro país como la farmacia comunitaria privada es ser los responsables de que los usuarios de los medicamentos reciban no solo la medicación correcta, lo que implica un adecuado despacho y dispensación del medicamento. Según Mariño, catedrático de farmacia galénica de la Facultad de Farmacia de la Universitat de Barcelona indica que "... lo que si está claro es que no necesariamente todas las actividades de la farmacia hospitalaria son farmacia clínica y que es posible hacer actividades de farmacia clínica fuera del ámbito hospitalario" (Mariño, 1989).

La profesión farmacéutica se ha tenido que reinventar muchas veces a lo largo de la historia de la humanidad, pasar de ser un preparador de medicamentos a un ámbito más clínico y en la actualidad orientarse hacia brindar distintos servicios que optimizan los resultados terapéuticos para usuarios de medicamentos individuales, que independientemente del suministro de un medicamento o relacionado con la dispensación del mismo, se pueda brindar una atención farmacéutica cada vez más integral. Bien lo decía Mariño (Mariño, 1989) hace ya 30 años, palabras que siguen vigentes en nuestra contexto actual:

"De esta forma, si conseguimos superar la crisis de identidad que ha experimentado nuestra profesión, existen unas expectativas de proyección muy interesantes, que solo podrán materializarse si contamos con una preparación suficiente, iniciativas para llevarlas a cabo y capacidad e intención de aceptación de las responsabilidades inherentes al ejercicio de la farmacia como actividad clínica, independientemente de la actuación a nivel de farmacia, hospitales, laboratorios farmacéuticos, universidades o centros de asistencia primaria, por citar algunos de los ámbitos más claramente implicados, aunque sin excluir a otros no reseñados." 
El concepto de atención farmacéutica fue desarrollado por Brodie (Brodie, Parish, \& Poston, 1980) a partir de la definición de Mikeal y otros autores (Mikeal, Brown, Lazarus, \& Vinson, 1975) y tiene como principal referencia el artículo de Hepler y Strand titulado "Opportunities and responsibilities in Pharmaceutical Care" (Hepler \& Strand) donde definen la atención farmacéutica como "la provisión responsable de una terapia medicamentosa con el propósito de conseguir resultados definidos que mejoran la calidad de vida del paciente. Estos resultados son: (1) curación de una enfermedad, (2) eliminación o reducción de la sintomatología, (3) interrupción o enlentecimiento del proceso patológico o (4) prevención de una enfermedad o sintomatología."

Hepler y Strand y otros autores (Hepler, The future of pharmacy: Pharmaceutical Care, 1990) (Cipolle, Strand, \& P.C., 1999) hicieron referencia que el proceso de atención farmacéutica se establece como una cooperación con el paciente y otros profesionales en el diseño, implementación y siguimiento del plan terapéutico, que producirá resultados terapéuticos específicos para el paciente, a partir de tres funciones prinicpales: (1) la identificación de los problemas relacionados con los medicamentos (PRM) potenciales y reales, (2) la resolución de PRM reales y (3) la prevención de PRM potenciales. En 1993, la OMS (Organización Mundial de la Salud) definió en Tokio (WHO, 1999) la atención farmacéutica: "el compendio de las actitudes, comportamientos, compromisos, inquietudes, valores éticos, funciones, conocimientos, responsabilidades y destrezas del farmacéutico en la prestación de la farmacoterapia, con el objetivo de lograr resulados terapéuticos definidos en la salud y calidad de vida del paciente." (Hepler, The future of pharmacy: Pharmaceutical Care, 1990) Se puede afirmar que la atención farmacéutica es un concepto de práctica asistencial, una nueva área del ejercicio profesional en la que el usuario del medicamento es el principal beneficiario de las acciones e intervenciones del farmacéutico.

Lamonitorización de resultadosterapéuticos(TOM, por su siglas en inglés Therapeutic Outcomes Monitoring) es una metodología desarrollada para evaluar la intervención farmacéutica en relación con los resultados terapéuticos de la medicación e implementar intervenciones de la gestión integral de la farmacoterapia por parte de los farmacéuticos a nivel asistencial (hospitalario o comunal). Por lo que el éxito de la implementación de nuevos servicios en atención farmacéutica deben estar soportados en un equilibrio entre los costes y los resultados que produce. Estos resultados deberán ser tanto económicos, clínicos como humanitarios (desde el punto de vista de la integralidad) y deberán abarcar una amplia clasificación en terminos de indicación terapéutica, efectividad, seguridad y cumplimiento (adherencia terapéutica).

En 1999 con la presentación de resultados de intervención farmacéutica con más de 12000 pacientes motivó a las aseguradoras a iniciar con la remuneración de los servicios de atención farmacéutica (Hepler, The future of pharmacy: Pharmaceutical Care, 1990). En 1997 Johnson y Bootman estimaron el impacto económico de la prestacion de la atención farmacéutica en el ambito ambulatorio en los Estados Unidos, concluyendo que la intervención farmacéutica evitaba la ocurrencia de los resultados terapéuticos negativos asociados al uso de los medicamentos (RNM) entre un 53-63\% y conseguía una reducción de 45,6 billones de dólares en costes directos de atención primaria en salud (Johnson \& Bootman, 1997).

La Medicare Prescription Drug, Improvement and Modernization Act (MMA, 2003) en los Estados Unidos incorporó una serie de requisitos para los servicios de atención farmacéutica denominado Medication Therapy Management (MTM) (Teagarden, 2006):

- Realizar u obtener las evaluaciones necesarias del estado de salud del paciente.

- Diseñar un plan de gestión farmacoterapéutico.

- Seleccionar, iniciar, modificar o administrar/ aplicar la farmacoterapia.

- Llevar a cabo el seguimiento y la evaluación 
de la respuesta del paciente a la farmacoterapia incluyendo la seguridad y la efectividad.

- Efectuar un análisis exhaustivo de la medicación para identificar, resolver y prevenir problemas relacionadso con los medicamentos, incluidos los eventos adversos de los mismos.

- Documentar la atención prestada y comunicar la información esencial a otros proveedores de la atención primaria de la salud del paciente.

- Proporcionar formación verbal y de habilidades encaminadas a mejorar la compresión del paciente y el uso adecuado de sus medicamentos.

- Facilitar información, servicios de apoyo y recursos diseñados para mejorar la adherencia de los pacientes a sus regímenes terapéuticos.

En Costa Rica, se ha generado un desarrollo académico de la atención farmacéutica desde los años 2000 desde la Universidad de Costa Rica (Facultad de Farmacia y el Centro de Nacional de Información de Medicamentos) en los que muchos colegas como la Dra. Victoria Hall y colaboradores han impulsado los servicios integrales de atenión farmacéutica que a nivel de los graduados de esta institución académica con 122 años y en multiples investigaciones publicadas, en la Caja Costarricense de Seguridad Social en los últimos 20 años han generado programas de atención farmacéutica integrando al profesional en farmacia más allá de la dispensación y despacho de medicamentos y equipos médicos, y se han orientado también a brindar seguimiento farmacoterapéuticos, consulta farmacéutica (por referencia a especialista), atención domiciliar e intervención a nivel comunitario, esto ha transformado las funciones y labores de los profesionales en farmacia a nivel clínico hospitalario. A nivel privado estamos aún muy en "pañales" se han desarrollado iniciativas como las que desarrolla el Dr. Javier León con su consulta farmacéutica privada que en los últimos años se ha enfocado en el tratamiento de personas con dolor y en estado terminal, recientemente con pacientes diabéticos, las primeras iniciativas se dieron poco antes del año 2007 en una farmacia privada (a cargo del Dr. Luis Carlos Monge Bogantes) se empesaron a dar los primeros pasos con los servicios privados de atención farmacéutica en el país, iniciativas que derivaron en el año 2010 a recibir el premio nacional Hans B. Raven otorgado por la Junta Directiva del Colegio de Farmacéuticos de Costa Rica, por destacarse por ser de los pioneros a nivel asistencial farmacéutico privado con iniciativas del desarrollo del consultorio privado en una farmacia comunitaria independiente que años después se fue especializando en atendión directa a pacientes con tratamientos de infertilidad, tratamientos biológicos modernos, promoción de la salud por medio de la inmunización (campañas de vacunación), entre otros productos especializados. A nivel privado también se ha destacado el Dr. José Miguel Chaverri como especialista certificado en farmacoterapia.

Los beneficios para los usarios de medicamentos de contar con un farmacéutico de confianza y que este le brinde servicios especializados de atención farmacéutica por medio de un consultorio privado puede beneficiar directamente a los pacientes polimedicados (que toman tres o más de tres medicamentos), que padezcan de una enfermedad crónica o que tengas varias comorbilidades y que además tenga una falla terapéutica (mala adherencia, poca efectividad, problemas de seguridad por el uso de sus tratamientos medicamentosos), podrán obtar a ser candidatos para se atendidos de forma integral y mejorar su caldidad de vida y poder usar de forma más eficaz, racional y segura sus medicamentos.

"Asimismo, cada vez se tiene más conciencia de que facilitar simplemente el medicamento a los usuarios no es suficiente para alcanzar los objetivos del tratamiento. Para abordar estas necesidades relacionadas con los medicamentos, losfarmacéuticosestánasumiendocada vez mayor responsabilidad en los resultados derivados de su uso, y están mejorando su práctica profesional para ofrecer a los pacientes mejores servicios en lo que respecta el uso" (OMS, 2011). 
Brindando servicios que favorescan un mayor beneficio de los tratamientos previamente establecidos, trabajando en conjunto con los prescriptores y demás personal de salud que atiende al paciente, integrando una gestión de la farmacovigilancia en la determinación de los riesgos asociados a la seguridad del paciente y evaluando la efetividad de los tratamientos, estableciendo un seguimiento farmacoterapéutico personalizado y mejorando así la adherencia terapéutica del paciente será como puede obtener los beneficios de la consulta farmacéutica a nivel privado.

En la UCIMED, estamos desarrollando este año 2019 la implementación de un consultorio farmacéutico privado para ofrecer una atención farmacéutica personalizada a la comunidad universitaria y la población que requiera más de estos nuevos servicios de atención. Se emplearan sistemas de dosificación personalizado (SPD) cuando el usuario del medicamento lo requiera según las condiciones propias de cada individuo atendido y de las diversas metodologías de intervención farmacéutica. Es importante recalcar la confidencialidad de los datos suministrados por el usuario del servicio de atención farmacéutica y del trato humanizado que se le brindará en el abordaje de cada uno de los casos clínicos que se podrán abordar cada caso en la consulta farmacéutica.

Los sistemas personalizados de dosificación permiten al farmacéutico individualizar la farmacoterapia de cada usuario y adaptarla a las necesidades específicas que tiene cada individuo, evaluando de previo interacciones farmacológicas, errores de medicación, adherencia terapéutica e interviniendo en la educación sanitaria del usuario y su red de cudido (en caso de necesitarlo), un sistema que permite mejorar mucho la adherencia terapéutica y facilitar el acceso seguro y eficaz de los medicamentos que esta utilizando un paciente; este es un ejemplo claro de uno de los beneficios de los pacientes al solicitar los servicios de atención farmacéutica especalizada en la consulta privada.

\section{Bibliografía:}

Francke, D. (1970). Pharmacy's new emphasis. Drug Intell Clin Pharm, num. 4, pag. 231.

Giráldez, J. (1985). Farmacia Clínica y Farmacovigilancia hospitalaria y comunitaria. Circ. Farm, núm 287, páginas 135-148.

Mariño, E. (1989). Farmacia Clínica y Universidad. El farmacéutico, núm. 72, páginas 39-57.

Brodie, D., Parish, P., \& Poston, J. (1980). Societal needs for drugs and drug related services. Am. J. Pharm. Ed. vol. 44, pag. 276-278.

Mikeal, R. L., Brown, T. P., Lazarus, H., \& Vinson, M. (1975). Quality of pharmaceutical care in hospitals. Am. J. Hosp. Pharm. vol. 32, pag. 567-574.

Hepler, C., \& Strand, L. (s.f.). Opportunities and responsibilities in pharmaceutical care. Am. J. Hosp. Pharm. vol. 47, pag. 533-543.

Hepler, C. (1990). The future of pharmacy: Pharmaceutical Care. Am. Pharm. vol.30, october, pag. 583-589.

Cipolle, R., Strand, L., \& P.C., M. (1999). El ejercicio de la atención farmacéutica. Madrid: Mc Graw-Hill.

Johnson, J., \& Bootman, J. L. (1997). Drug related morbidity and mortality and the economic impact of pharmaceutical care. Am. J. Health-Syst. Pharm. Vol. 54 , pag. 554-558.

WHO. (1999). El papel del farmacéutico en el sistema de atención de salud. Tokio: Informe en Pharm. Care Esp. Vol. 1 (supl. 3), pag. 207-211.

Teagarden, J. R. (2006). Medication Therapy Management and the Medicare prescription drug benefit. Hosp. Pharm. Vol. 41, pag. 88-90. 


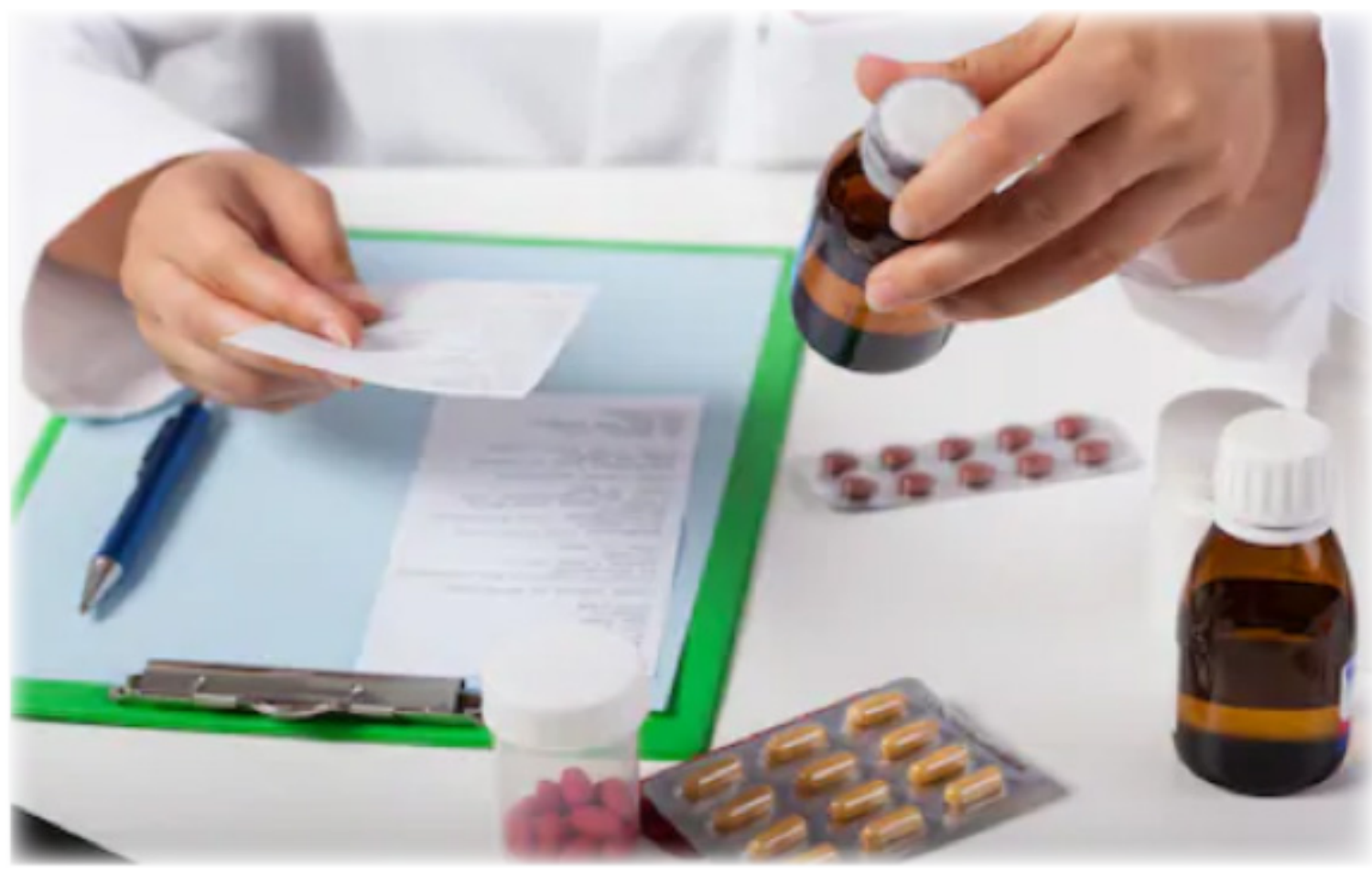

Consulta Farmacéutica No. 2

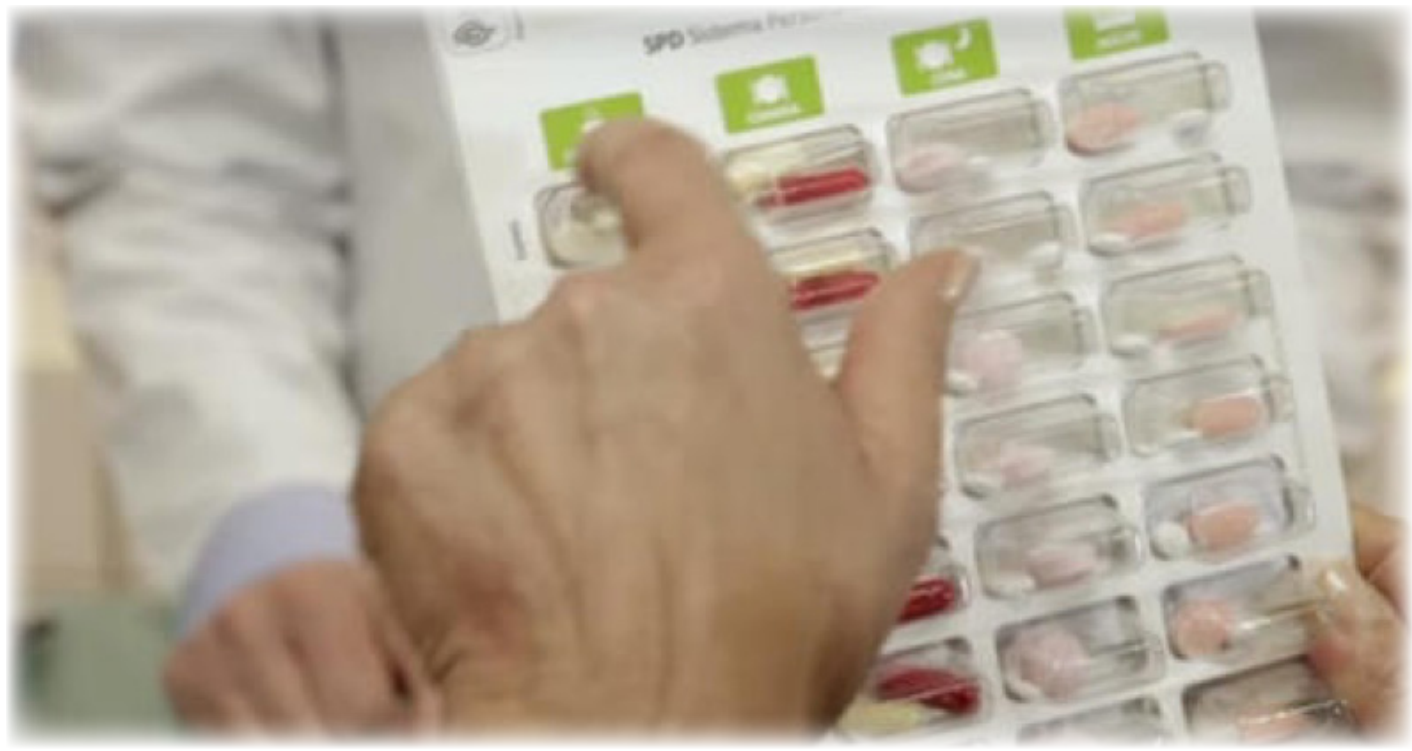

Sistema de Dosificación Personalizada, SPD en la consulta farmacéutica.
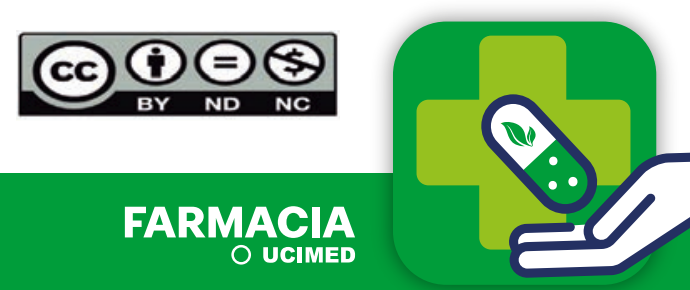